Research Article

\title{
An Approach to Improve SSD through Skip Connection of Multiscale Feature Maps
}

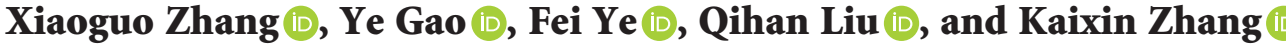 \\ School of Instrument Science and Engineering, Southeast University, Nanjing 210096, China \\ Correspondence should be addressed to Xiaoguo Zhang; xgzhang@seu.edu.cn
}

Received 31 October 2019; Accepted 24 January 2020; Published 16 March 2020

Academic Editor: Cornelio Yáñez-Márquez

Copyright (c) 2020 Xiaoguo Zhang et al. This is an open access article distributed under the Creative Commons Attribution License, which permits unrestricted use, distribution, and reproduction in any medium, provided the original work is properly cited.

\begin{abstract}
SSD (Single Shot MultiBox Detector) is one of the best object detection algorithms and is able to provide high accurate object detection performance in real time. However, SSD shows relatively poor performance on small object detection because its shallow prediction layer, which is responsible for detecting small objects, lacks enough semantic information. To overcome this problem, SKIPSSD, an improved SSD with a novel skip connection of multiscale feature maps, is proposed in this paper to enhance the semantic information and the details of the prediction layers through skippingly fusing high-level and low-level feature maps. For the detail of the fusion methods, we design two feature fusion modules and multiple fusion strategies to improve the SSD detector's sensitivity and perception ability. Experimental results on the PASCAL VOC2007 test set demonstrate that SKIPSSD significantly improves the detection performance and outperforms lots of state-of-the-art object detectors. With an input size of $300 \times 300$, SKIPSSD achieves $79.0 \% \mathrm{mAP}$ (mean average precision) at $38.7 \mathrm{FPS}$ (frame per second) on a single $1080 \mathrm{GPU}, 1.8 \%$ higher than the MAP of SSD while still keeping the real-time detection speed.
\end{abstract}

\section{Introduction}

The object detection algorithms based on deep learning could be roughly divided into two categories: based on region proposals and based on end to end. As known, the former models generally include R-CNN [1], Fast R-CNN [2], Faster R-CNN [3], and R-FCN [4], which firstly generate a category-independent set of region proposals for subsequent feature extraction and classification. The two most popular latter models based on end to end are YOLO (You Only Look Once) [5] and SSD (Single Shot MultiBox Detector) [6], which need setting the default box, training the network, and establishing the relationship of the prior box, default box, and ground truth box.

The two-stage methods such as SPP-net [7], Fast R-CNN [2], and Faster R-CNN [3], generally only use the last layer as the prediction layer, but the layer with the fixed receptive field size is not suitable for both too larger and smaller object detections. SSD innovatively uses the pyramid feature hierarchy of ConvNet and combines predictions from multiple feature maps with different resolutions to deal with the scale variation problem for object detector. Generally speaking, SSD is not only able to achieve real-time object detection but also known for its high detection accuracy. On the PASCAL VOC 2007 test [8], SSD achieves $77.2 \% \mathrm{mAP}$ at the speed of 46 FPS with the input size $300 \times 300$ using a single NVIDIA Titan X GPU [6]. However, the linkages between multiscale prediction layers of SSD are not fully considered, and the low-level feature maps lack enough semantic information for small object detection; thus, SSD shows poor performance on small object detection [9]. As shown in Figure 1, some small objects, for example boats in the red box, are not detected by SSD.

To deal with the problem that SSD shows poor performance on small object detection and to maintain a satisfactory detection speed at the same time, we adopt a novel skip connection of multiscale feature maps to SSD, and the overall architecture is illustrated in Figure 2. The main contributions are summarized as follows: (1) SKIPSSD, an improved SSD with a novel skip connection of multiscale feature maps, is proposed to enhance the semantic information and the details of the prediction layers through 


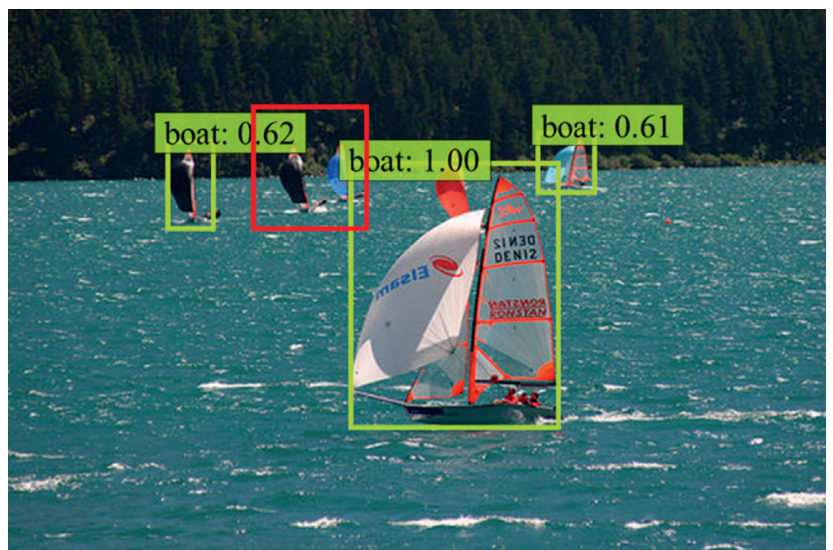

FIgURE 1: The framework of our SKIPSSD. SKIPSSD skippingly fuses high-level and low-level feature maps to enhance semantic information of the model.

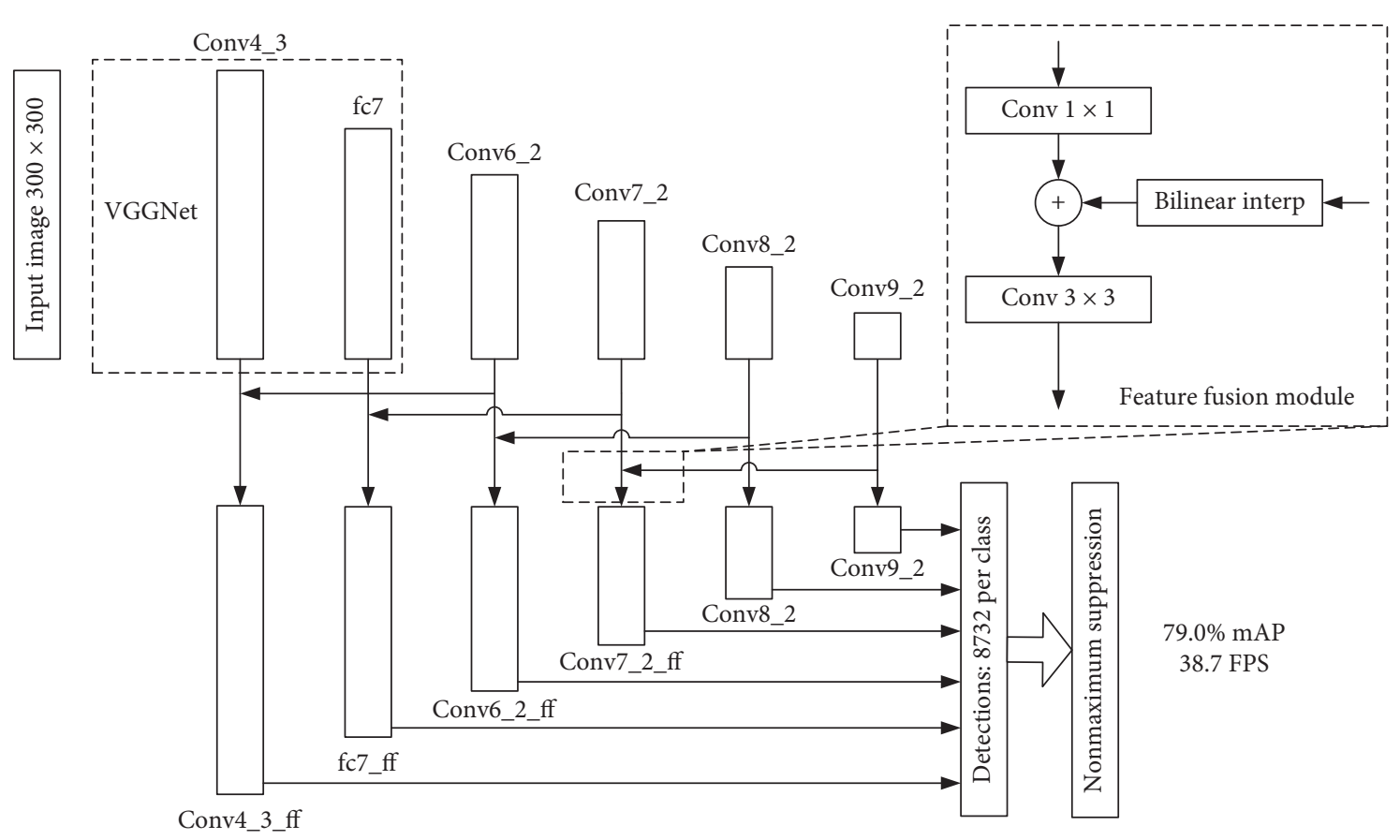

FIGURE 2: The framework of our SKIPSSD. SKIPSSD skippingly fuses high-level and low-level feature maps to enhance semantic information of the model.

skippingly fusing high-level and low-level features; (2) six multiscale feature maps fusion structures over the SSD network, and two feature fusion modules and multiple fusion strategies are designed to investigate the optimal feature fusion framework; (3) experiments on the PASCAL VOC 2007 test set are conducted to compare the performance of SKIPSSD with other state-of-the-art object detectors.

The experimental results demonstrate that SKIPSSD significantly improves the detection performance and outperforms a lot of state-of-the-art object detectors. With an input size of $300 \times 300$, SKIPSSD achieves $79.0 \% \mathrm{mAP}$ (mean average precision) at 38.7 FPS (frame per second) on a single 1080 GPU, $1.8 \%$ higher than the mAP of SSD while still keeping the real-time detection speed.

\section{Methodology}

2.1. Related Work. In the field of object detection, image pyramids are often used to solve the degradation of detection performance caused by the change of object scale. However, such kind of algorithms is very time consuming. SSD innovatively uses a ConvNet's pyramidal feature hierarchy and combines predictions from multiple layers with different scales, mitigating the problem of object scale change in certain degree [6]. However, the linkages between multiscale prediction layers of SSD are not fully considered, and the low-level feature maps lack enough semantic information for small object detection; thus, SSD shows poor performance on small object detection [9]. 
In order to deal with the abovementioned problem of SSD, DSSD (Deconvolutional Single Shot Detector) [10] uses Resnet-101 [11] in place of VGG used in SSD and adds deconvolutional layers to introduce large-scale context. Although DSSD improves the performance of small object detection of SSD, its detection speed is much slower than SSD, and it is not able to realize real-time detection. After that, much has been done to balance accuracy and speed for small object detection of SSD. RSSD [12] adopts weightsharing strategy between different layers to SSD and improves the accuracy by $0.8 \%$ with the speed dropping to 35 FPS because of the increase of computational complexity. FSSD [9] uses a lightweight and efficient feature fusion module and achieves $78.8 \% \mathrm{mAP}$ on the VOC2007 test set at 65.8 FPS, outperforming RSSD300 on both accuracy and speed. Feature-Fused SSD300 [13] simply conducts elt_sum function between Conv4_3 and Conv5_3 of SSD and achieves $78.9 \% \mathrm{mAP}$, which is little higher than FSSD. Combining the advantages of the two-stage and one-stage methods, RefineDet [14] uses the ARM module to reduce the search space, transfers the features from the ARM to ODM module through the TCB connection module, and achieves $81.8 \% \mathrm{mAP}$ at $40.3 \mathrm{FPS}$ with an input size $512 \times 512$, surpassing DSSD513. To further improve the accuracy of SSD, DES (Detection with Enriched Semantics) [15] not only introduces the segmentation module to obtain the mask, which objectively enhances the semantic information of the shallow features, but also introduces the global activation module to enhance the semantic information of the highlevel features. DES512 boosts the mAP on the VOC2007 test set to $81.7 \%$ at $31.7 \mathrm{FPS}$. The accuracy of DES512 is improved at the cost of speed.

The abovementioned algorithms improve the detection accuracy of SSD. However, their inference time increase a lot. Overall speaking, it is of great value to obtain high precision object detection performance with satisfactory real time performance.

2.2. Multiscale Feature Fusion Structure Design. Many works have led to the discovery that the features from different layers of the network are complementary, and integrating the multiscale features can benefit the multiscale object detection $[9,10,16]$. More specifically, the very invariance properties of the DCNN model make the high-level feature maps of DCNN learn abstract features of data well which is good for object recognition but show side effect to object location, and the low-level feature maps contain rich spatial structural details which are beneficial to locate objects. Inspired by an encoder-decoder network U-Net [17], which uses the skip connection to associate low-level feature maps and high-level feature maps to realize the positioning at the pixel level, we adopt the idea of skip connection to SSD to enhance semantic information. In this paper, we design and evaluate a series of multiscale feature maps fusion structures over SSD to explore the optimal fusion structure: multiscale prediction feature maps skip connection (SKIPSSD), part skip connection (Part-SKIPSSD), bidirectional skip connection (Bi-SKIPSSD), skip connection with partial feature maps of base network (Base-SKIPSSD), adjacent connection (AdjacentSSD), and multiscale prediction feature pyramid network (FPNSSD).

Figure 3(a) shows the skip connection between the multiscale prediction feature maps, where SKIPSSD upsamples Conv9_2 to fuse with Conv7_2 to get Conv7_2_ff, upsamples Conv8_2 to fuse with Conv6_2 to get Conv6_2_ff, upsamples Conv7_2 to fuse with fc7 to get fc7_ff, and upsamples Conv6_2 to fuse with Conv4_3 to get Conv4_3_ff. The multiscale prediction feature maps skipconnection network is called SKIPSSD in this paper. Figure 3(b) shows the structure of the prediction layer of SKIPSSD, Conv8_2, and Conv9_2, and the fused feature maps Conv4_3_ff, fc7_ff, Conv6_2_ff, and Conv7_2_ff are used as the multiscale prediction layers.

As shown in Figure 4(a), the structure of Part-SKIPSSD is described. Compared with SKIPSSD, Part-SKIPSSD removes the feature fusion of Conv7_2 layer and only uses the Conv4_3_ff, fc7_ff, and Conv6_2_ff and Conv7_2, Conv8_2, and Conv9_2 of the original SSD as the multiscale prediction feature maps.

The structure in Figure 5(a) is called Bi-SKIPSSD in this paper. Compared with SKIPSSD, Bi-SKIPSSD adds the feature fusion of layers Conv8_2 and Conv9_2, respectively. Conv6_2, which undergoes convolution and pooling layers, is then merged with Conv8_2 to generate Conv8_2_ff. Similarly, Conv7_2, which undergoes convolution and pooling layers, is then merged with Conv9_2 to generate Conv9_2_ff. The Figure 5(b) shows that Bi-SKIPSSD uses Conv4_3_ff, fc7_ff, Conv6_2_ff, Conv7_2_ff, Conv8_2_ff and Conv9_2_ff as the multiscale prediction feature maps.

Different from the abovementioned three networks, Base-SKIPSSD in Figure 6 conducts skip connection between the layers in VGG base network and prediction layers. Conv4_1, which undergoes convolutional and pooling layers, is then merged with Conv4_3 to generate Conv4_3_ff, and the latter fc7, Conv6_2, Conv7_2, Conv8_2, and Conv9_2 are merged with their corresponding basic feature layers in a similar way. The fusion feature maps Conv4_3_ff, fc7_ff, Conv6_2_ff, Conv7_2_ff, Conv8_2_ff, and Conv9_2_ff act as the multiscale prediction feature maps.

The structure in Figure 7(a) is called AdjacentSSD. Compared with SKIPSSD, AdjacentSSD conducts adjacent connection instead of skip connection. Layer fc7, which undergoes the upsampling layer, is merged with Conv4_3 to generate Conv4_3_ff, and the latter layers Conv6_2, Conv7_2, Conv8_2, and Conv9_2 are merged with their adjacent layers in a similar way. The Figure 7(b) shows that AdjacentSSD uses Conv4_3_ff, fc7_ff, Conv6_2_ff, Conv7_2_ff, Conv8_2_ff, and Conv9_2 as the multiscale prediction feature maps.

The structure in Figure 8(a) is called FPNSSD. Different from AdjacentSSD, in which the high-level layer Conv9_2 is only fused with adjacent layer Conv8_2 to generate Conv8_2_ff, FPNSSD adopts a top-down architecture with lateral connections called FPN [16], shown in Figure 9, and the high-level layer Conv9_2 is fused layer by layer and contributes to the generation of Con8_2_ff, Conv7_2_ff, and so on. The Figure 8(b) shows that FPNSSD uses Conv4_3_ff, 


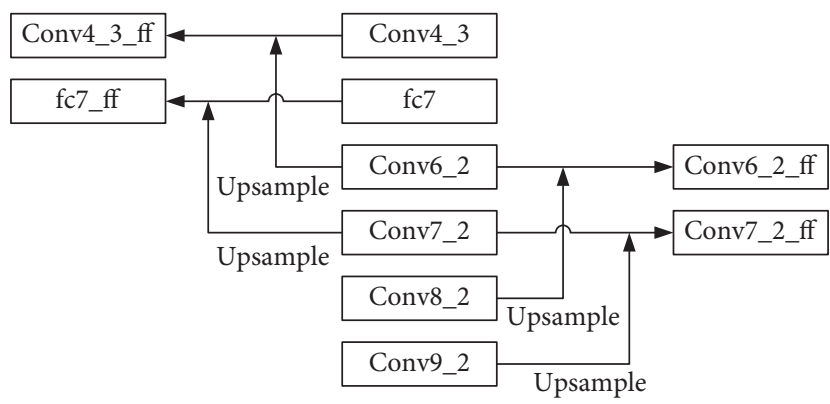

(a)

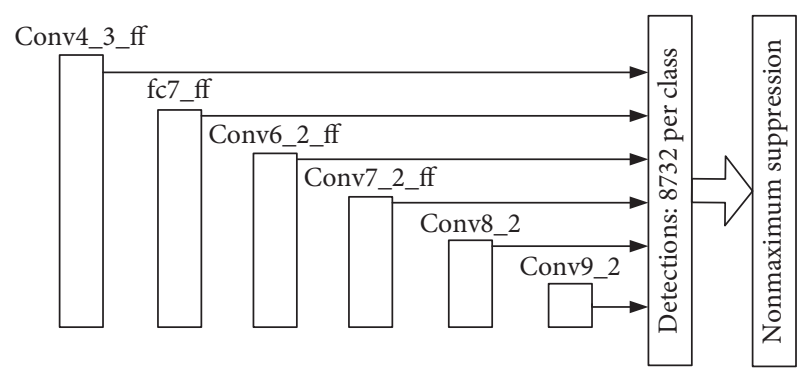

(b)

Figure 3: Multiscale prediction feature maps skip connection (SKIPSSD). (a) Skip connection structure. (b) Multiscale prediction layers.

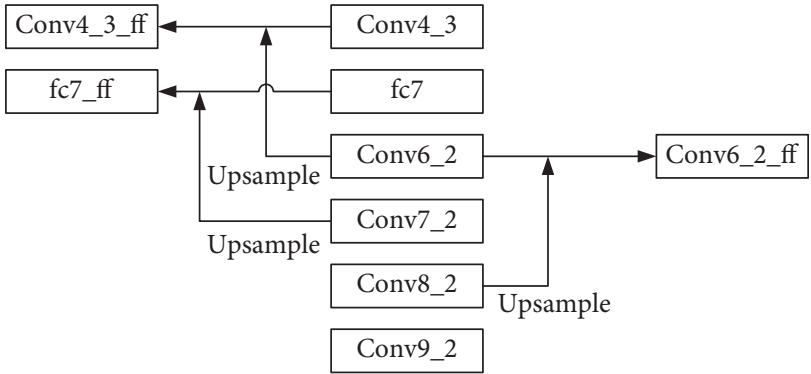

(a)

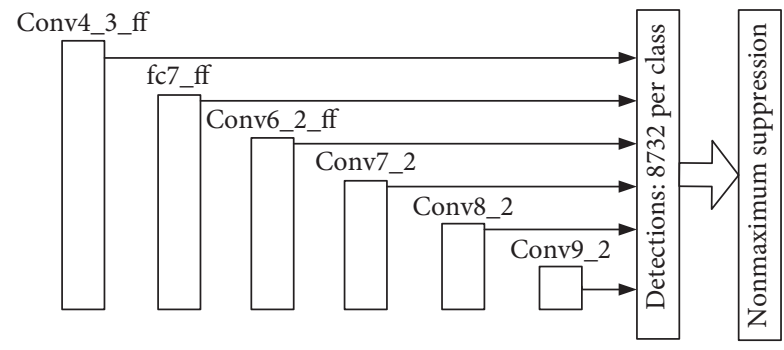

(b)

Figure 4: Multiscale prediction feature maps part skip connection (part-SKIPSSD). (a) Part skip connection structure. (b) Multiscale prediction layers.

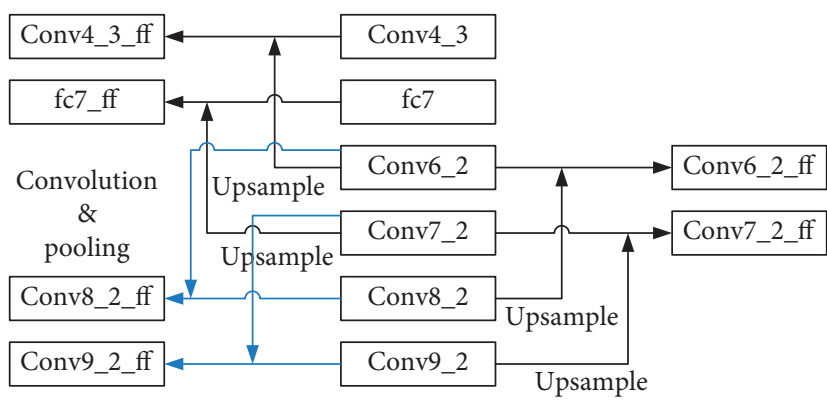

(a)

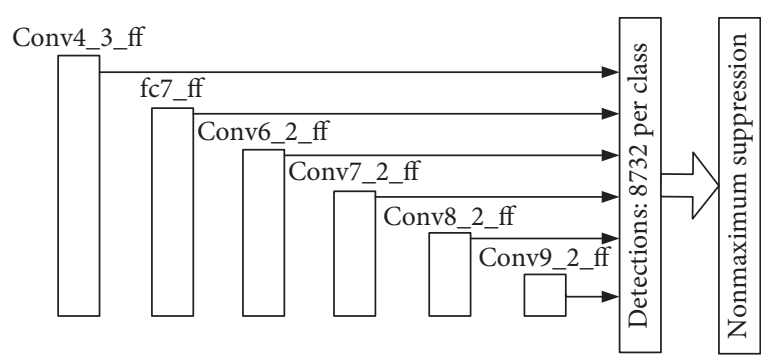

(b)

FIGURE 5: Multiscale prediction feature maps bidirectional skip connection (Bi-SKIPSSD). (a) Bidirectional skip connection structure. (b) Multiscale prediction layers.

fc7_ff, Conv6_2_ff, Conv7_2_ff, Conv8_2_ff, and Conv9_2 as the multiscale prediction feature maps.

The abovementioned six multiscale feature maps fusion structures over SSD are analysed and evaluated on the PASCAL VOC2007 test set in Section 3.4 to explore the optimal multiscale feature maps fusion structure.

2.3. Feature Fusion Module Design. In this paper, two feature fusion modules are designed for high-level and low-level feature fusion, and the fusion effect is compared in our experiments. Concat and eltsum are two common methods of feature fusion. Concat operation is channel concatenation of two feature maps. In Caffe, there are three operations of the Eltwise layer: product (dot product), sum (add or subtract), and max (take the large value), and the sum operation is selected in this paper.

As shown in Figure 10, fusion module $a$ firstly upsamples the high-level feature map to generate high-level feature map_up, which undergoes $3 \times 3$ convolutional layer and relu activation function to obtain high-level feature map_fused. In addition, the low-level feature map, which undergoes $3 \times 3$ convolutional layer and relu activation function, is transformed into low-level feature map_fused. Then, concat or eltsum function is applied to conduct feature fusion between low-level feature map_fused and high-level feature map_fused to obtain the high-low-level feature map concat/ sum. Finally, the high-low-level feature map concat/sum, 


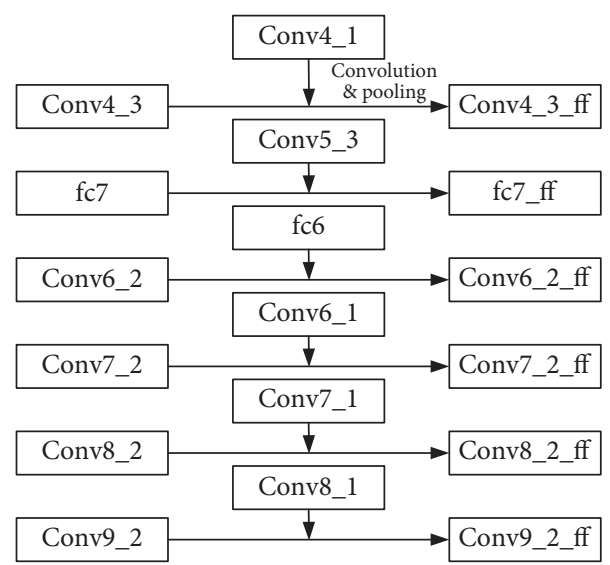

(a)

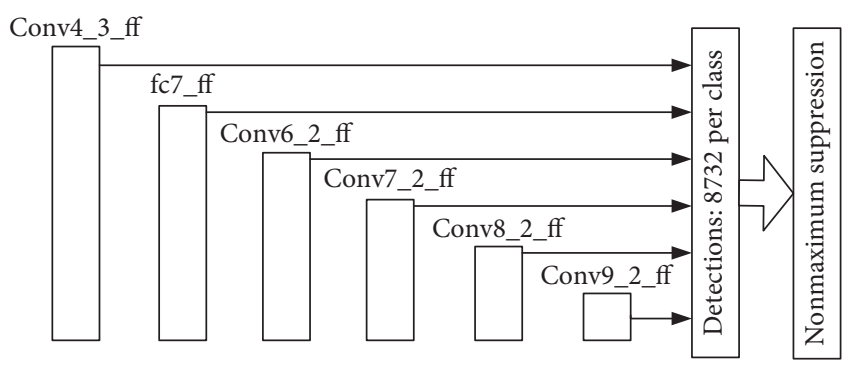

(b)

FIGURE 6: Skip connection with partial feature maps of base network (base-SKIPSSD). (a) Skip connection structure with partial feature maps of base network. (b) Multiscale prediction layers.

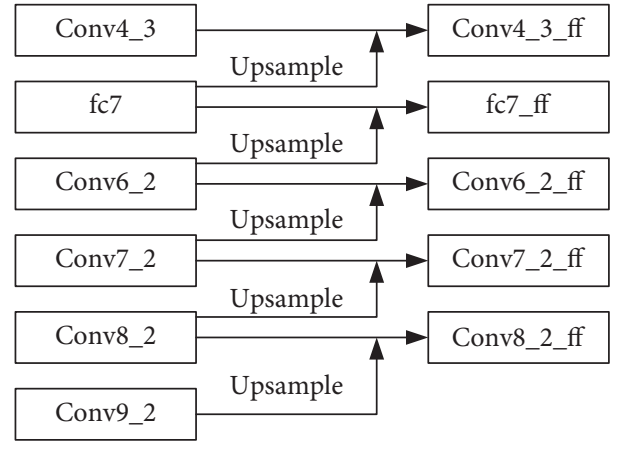

(a)

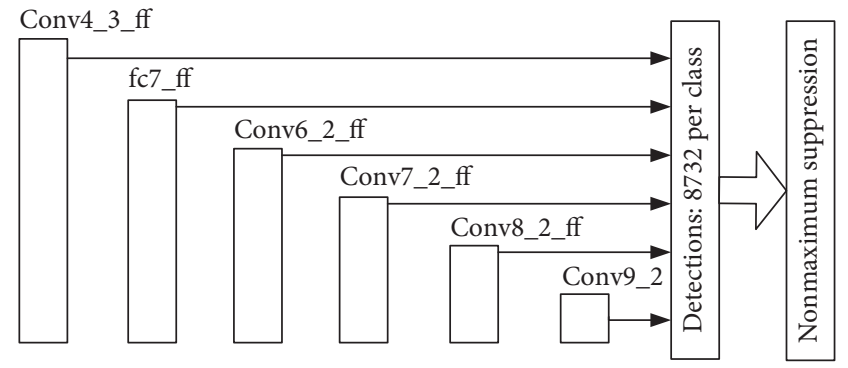

(b)

FIGURE 7: Multiscale prediction feature maps adjacent connection (adjacentSSD). (a) Multiscale prediction feature maps adjacent connection structure. (b) Multiscale prediction layers.

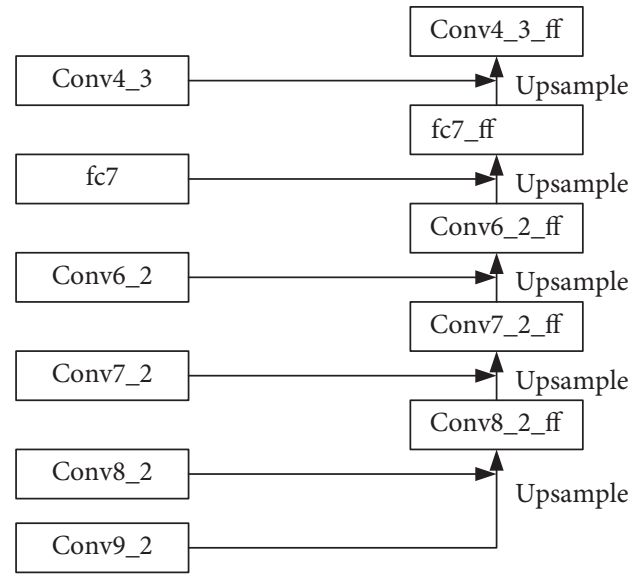

(a)

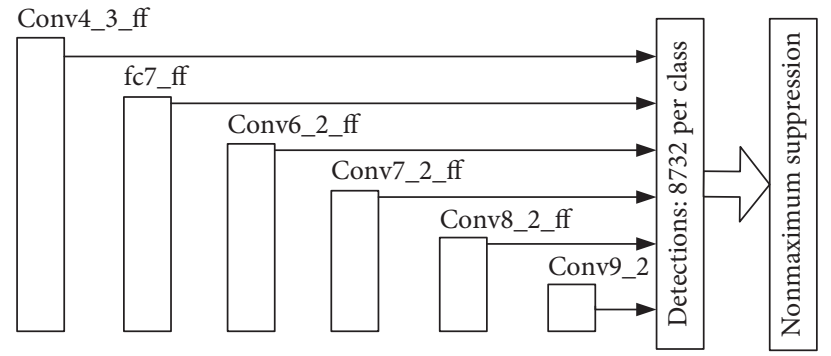

(b)

FIGURE 8: Multiscale prediction feature pyramid network (FPNSSD). (a) Multiscale prediction feature pyramid network structure. (b) Multiscale prediction layers. 


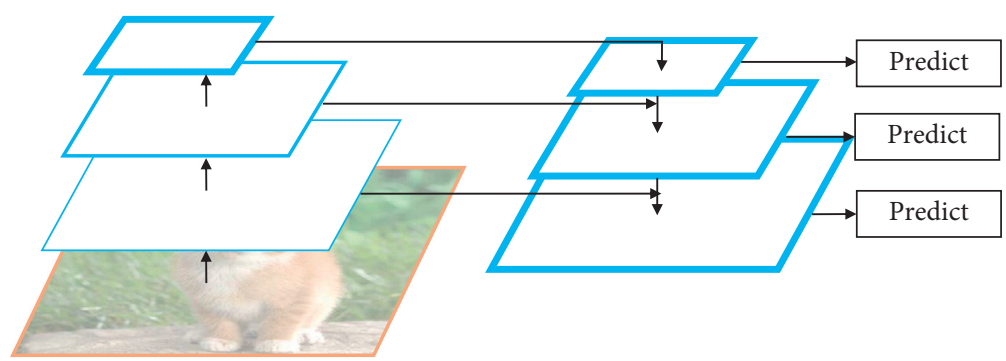

FIGURE 9: Feature pyramid network.

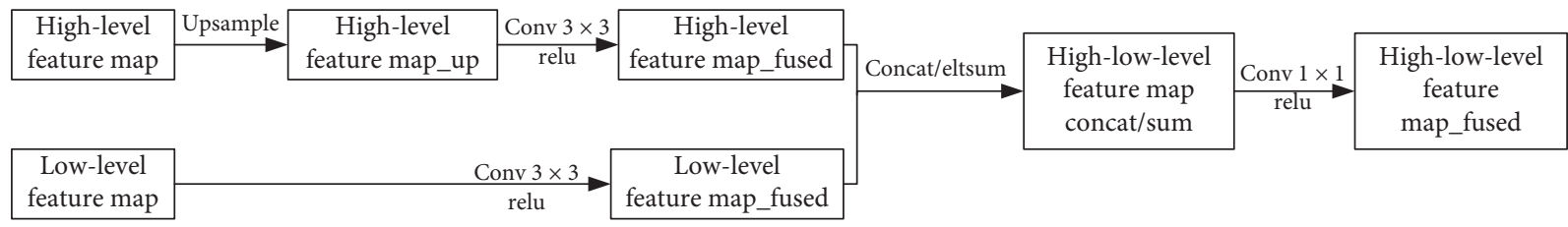

Figure 10: Fusion module $a$.

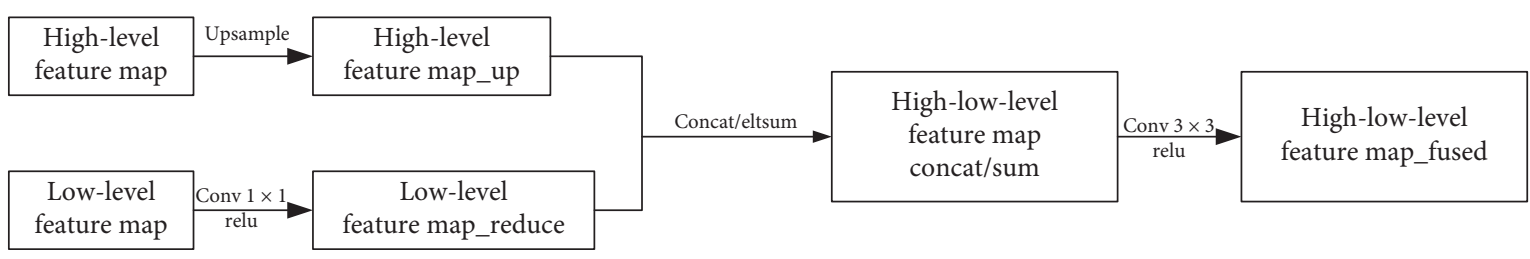

Figure 11: Fusion module $b$.

which undergoes a $1 \times 1$ convolutional layer to reduce channel dimensions, is activated with the relu activation function to obtain the prediction feature map high-low-level feature map_fused.

Compared with fusion module $a$ in Figure 10, the fusion module $b$ in Figure 11 is briefer. Firstly, fusion module $b$ upsamples the high-level feature map to generate high-level feature map_up and transform low-level feature map into low-level feature map_reduce through a $1 \times 1$ convolutional layer. Then, concat or eltsum function is applied to conduct feature fusion between low-level feature map_reduce and high-level feature map_up to obtain the high-low-level feature map concat/sum. Finally, the high-low-level feature map concat/sum, which undergoes a $3 \times 3$ convolutional layer to reduce the aliasing effect, is activated with the relu activation function to obtain the prediction feature map high-low-level feature map_fused.

\section{Results and Discussion}

To evaluate the performance of the proposed improved SSD network and to find the optimal multiscale feature maps fusion framework, four types of test cases are designed in this paper:

(1) Compare the performance of SKIPSSD when using different feature fusion modules. Through this experiment, the most effective feature fusion module can be found.

(2) Compare the influence of different fusion strategies on SKIPSSD model performance. Through this
TABLE 1: Experimental hardware and software configurations.

\begin{tabular}{lc}
\hline Hardware and software & Profile \\
\hline CPU & Intel XeonE5-2620v4@2.10GHz \\
GPU & GeForce GTX 1080 \\
Operating system & Ubuntu 16.04 \\
Deep learning frame & Caffe \\
\hline
\end{tabular}

experiment, the most effective fusion strategy can be selected.

(3) Compare the effect of different upsampling methods on SKIPSSD model performance. Through this experiment, the most effective upsampling method can be selected.

(4) Compare the performance of SSD with different feature maps fusion structures. Through this experiment, the most effective feature fusion structure can be found.

The experimental hardware and software configurations are listed in Table 1. In order to evaluate the performance of SKIPSSD, the union of VOC2007 trainval and VOC2012 trainval is used as the training data, and the VOC2007 test as the test data. For fair comparison, the experiments are all based on VGG16, which is preprocessed as what is conducted in SSD, and SKIPSSD is trained in the same way as SSD. The parameter settings are listed in Table 2. The mAP and FPS are adopted as the metric for evaluating detection performance. 
TABLE 2: Training parameter settings.

\begin{tabular}{lc}
\hline Parameter & Size \\
\hline Input size & $300 \times 300$ \\
Number of iterations & 240,000 steps \\
Batch size & 16 \\
Initial learning rate & 0.0005 (it is divided by 10 in steps of 160,000, 200000, and 240,000) \\
Weight learning rate & 0.0005 \\
Momentum & 0.9 \\
\hline
\end{tabular}

TABLE 3: The effect of the feature fusion connection module on model performance.

\begin{tabular}{|c|c|c|c|c|c|c|}
\hline Model & Data & Pretrained model & Fusion module & Fusion method & FPS & mAP (\%) \\
\hline SSD & $07+12$ & VGGNet & $x$ & $x$ & 41.4 & 77.2 \\
\hline SKIPSSD & $07+12$ & VGGNet & $a$ & Eltsum & 32.3 & 78.1 \\
\hline SKIPSSD & $07+12$ & VGGNet & $b$ & Eltsum & 37.8 & 78.3 \\
\hline
\end{tabular}

TABLE 4: The effect of different fusion strategies on model performance.

\begin{tabular}{|c|c|c|c|c|c|}
\hline Model & Data & Pretrained model & Fusion model & $\mathrm{BN}$ & mAP (\%) \\
\hline SKIPSSD & $07+12$ & VGGNet & Concat & Used after all convolution layers of the fusion module & 77.9 \\
\hline SKIPSSD & $07+12$ & VGGNet & Eltsum & $x$ & 78.0 \\
\hline SKIPSSD & $07+12$ & VGGNet & Eltsum & Used after all convolution layers of the fusion module & 78.3 \\
\hline SKIPSSD & $07+12$ & VGGNet & Eltsum & Used only after the eltsum function & 78.4 \\
\hline
\end{tabular}

Conv4_3_fea_eltsum

\begin{tabular}{|c|}
\hline Operation: sum \\
\hline Input: conv6_2 \\
Type: deconvolution \\
deconv6-512 \\
(dilation = 2) \\
Output: deconv6_2 \\
\\
\\
Input: conv4_3 \\
Type: convolution \\
conv1-512 \\
Output: conv4_3_reduce \\
\end{tabular}

FIGURE 12: Configuration of sampling parameters on deconvolution and dilated convolution.
Conv6_2_fea_eltsum

\begin{tabular}{|c|}
\hline Operation: sum \\
\hline Input: conv7_2 \\
Type: deconvolution \\
deconv3-1024 \\
(dilation=3) \\
Output: deconv7_2 \\
\hline \\
Input: fc7 \\
Type: convolution \\
conv1-1024 \\
Output: fc7_reduce \\
\end{tabular}

\begin{tabular}{|c|}
\multicolumn{1}{|c|}{ Conv6_2_fea_eltsum } \\
\hline Operation: sum \\
\hline Input: conv8_2 \\
Type: deconvolution \\
deconv4-512 \\
(dilation = 3) \\
Output: deconv8_2 \\
\\
\\
Input: conv6_2 \\
Type: convolution \\
conv1-512 \\
Output: conv6_2_reduce \\
\end{tabular}

\begin{tabular}{|c|}
\hline Conv7_2_fea_eltsum \\
\hline Operation: sum \\
\hline Input: conv9_2 \\
Type: deconvolution \\
deconv5-256 \\
Output: deconv9_2 \\
\\
\\
Input: conv7_2 \\
Type: convolution \\
conv1-256 \\
Output: conv6_2_reduce \\
\end{tabular}

3.1. The Effect of Two Feature Fusion Modules on Model Performance. In order to find the optimal feature fusion module, SKIPSSD with different feature fusion modules are evaluated on the PASCAL VOC 2007 test, and the performance evaluated with an input size $300 \times 300$ is recorded in Table 3. In this experiment, the network of SKIPSSD is shown in Figure 3, and the BN (Batch Normalization) layer is added after all the convolutional kernels in the fusion module.

According to the results in Table 3, SKIPSSD with fusion module $a$ achieves $78.1 \%$ mAP, $0.9 \%$ higher than SSD, and the mAP of SKIPSSD with fusion module $b$ is $1.1 \% \mathrm{mAP}$ higher than SSD, demonstrating that skip connection of multiscale feature maps indeed improves the performance of SSD. Since SKIPSSD with fusion module $b$ outperforms fusion module $a$ on both accuracy and speed, the fusion module $b$ is chosen in this paper for high-low level feature fusion.

\subsection{The Effect of Fusion Strategies on Model Performance.} In this experiment, two aspects of the factors are compared: (1) concat and eltsum fusion methods; (2) full and partial use of BN layers. The experimental results are recorded in Table 4. In the experiment, the upsampling method is deconvolution and dilated convolution, and the fusion module is $b$. 

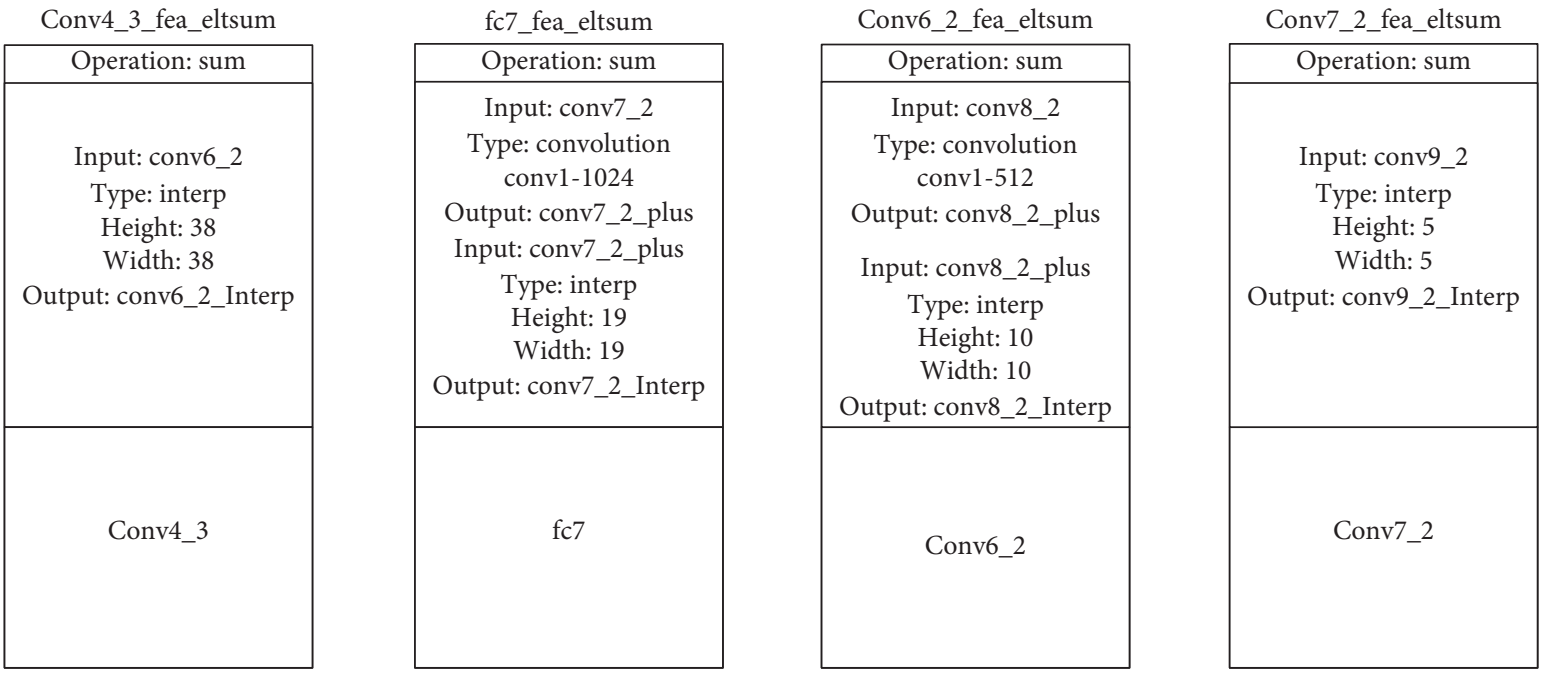

FIgURE 13: Configuration of sampling parameters on bilinear interpolation.

TABLE 5: Effect of different upsampling methods on model performance.

\begin{tabular}{lccccc}
\hline Model & Data & Pretrained model & Upsampling method & FPS & mAP (\%) \\
\hline SKIPSSD & $07+12$ & VGGNet & Deconvolution + dilated convolution & 36.8 & 78.4 \\
SKIPSSD & $07+12$ & VGGNet & Bilinear interpolation & 38.7 & 79.0 \\
\hline
\end{tabular}

TABLE 6: Effect of different feature fusion network structures on model performance.

\begin{tabular}{lcccc}
\hline Model & Data & Pretrained model & FPS & mAP (\%) \\
\hline SKIPSSD & $07+12$ & VGGNet & 38.7 & 79.0 \\
Part-SKIPSSD & $07+12$ & VGGNet & 39.2 & 78.7 \\
Bi-SKIPSSD & $07+12$ & VGGNet & 38.1 & 78.8 \\
Base-SKIPSSD & $07+12$ & VGGNet & 39.2 & 78.6 \\
AdjacentSSD & $07+12$ & VGGNet & 38.0 & 78.8 \\
FPNSSD & $07+12$ & VGGNet & 37.9 & 78.8 \\
\hline
\end{tabular}

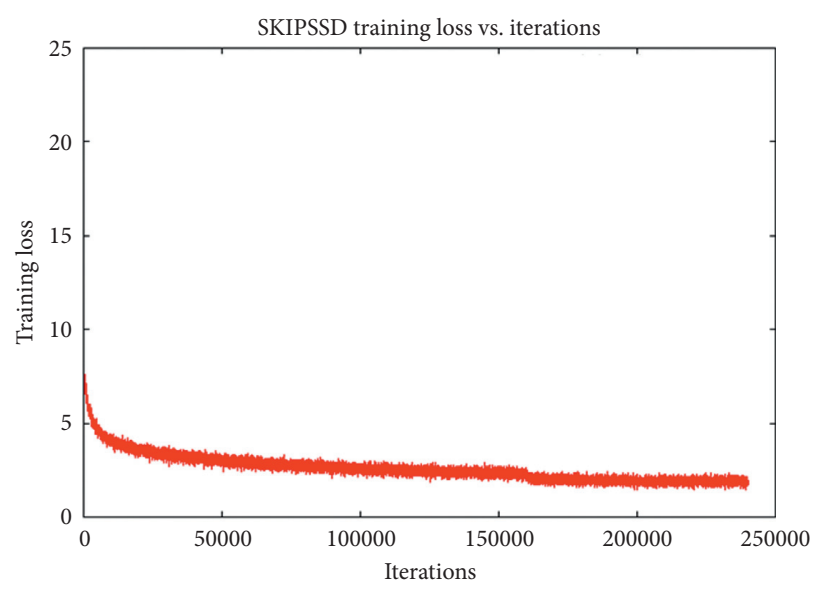

(a)

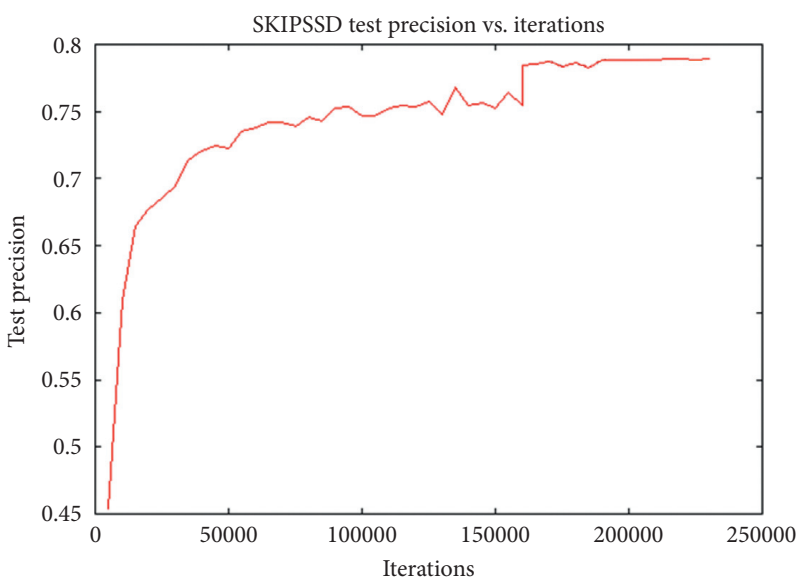

(b)

FIgURE 14: The loss and precision curves. (a) Iterations-training loss curve. (b) Iterations-test precision curve. 


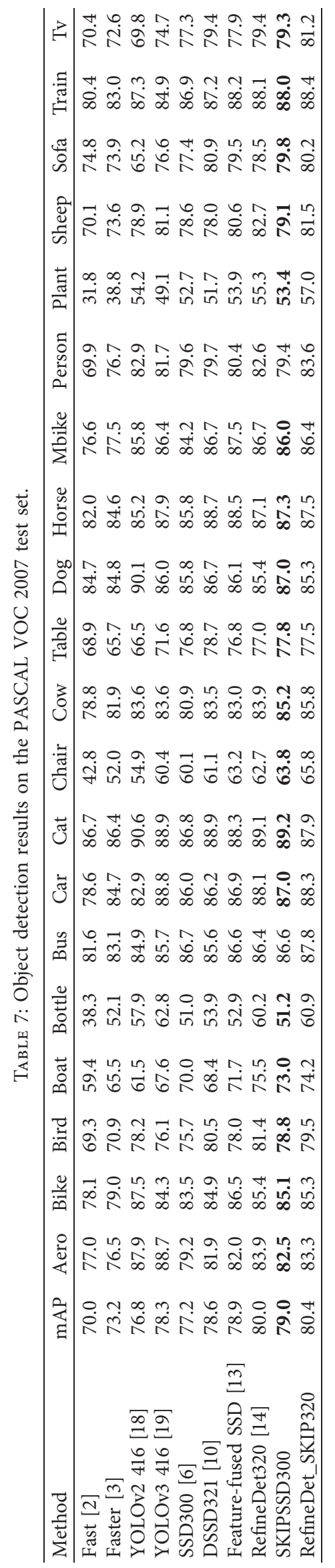


TABLE 8: Comparison of speed and accuracy on the PASCAL VOC2007 test dataset.

\begin{tabular}{|c|c|c|c|c|c|c|}
\hline Model & Data & Base network & mAP (\%) & FPS & GPU & Input size \\
\hline Faster R-CNN [3] & $07+12$ & VGGNet & 73.2 & 7 & Titan X & $\sim 600 \times 1000$ \\
\hline R-FCN [4] & $07+12$ & ResNet-101 & 79.5 & 9 & Titan X & $\sim 600 \times 1000$ \\
\hline YOLOv2 [18] & $07+12$ & VGGNet & 76.8 & 67 & Titan X & $416 \times 416$ \\
\hline YOLOv3 [19] & $07+12$ & VGGNet & 78.3 & 57.7 & Titan Xp & $416 \times 416$ \\
\hline SSD300 [6] & $07+12$ & VGGNet & 77.2 & 46 & Titan X & $300 \times 300$ \\
\hline DSSD321 [10] & $07+12$ & ResNet-101 & 78.6 & 9.5 & Titan X & $321 \times 321$ \\
\hline DSOD300 [20] & $07+12$ & DS/64-192-48-1 & 77.7 & 17.4 & Titan X & $300 \times 300$ \\
\hline RSSD300 [12] & $07+12$ & VGGNet & 78.5 & 35 & Titan X & $300 \times 300$ \\
\hline FSSD300 [9] & $07+12$ & VGGNet & 78.8 & 35 & Titan X & $300 \times 300$ \\
\hline RefineDet [14] & $07+12$ & VGGNet & 80.0 & 40.3 & Titan X & $320 \times 320$ \\
\hline SSD300 [6] & $07+12$ & VGGNet & 77.2 & 41.4 & 1080 & $300 \times 300$ \\
\hline RSSD300 [12] & $07+12$ & VGGNet & 78.5 & 34.8 & 1080 & $300 \times 300$ \\
\hline RefineDet [14] & $07+12$ & VGGNet & 80.0 & 36.0 & 1080 & $320 \times 320$ \\
\hline SKIPSSD300 & $07+12$ & VGGNet & 79.0 & 38.7 & 1080 & $300 \times 300$ \\
\hline RefineDet_SKIP & $07+12$ & VGGNet & 80.4 & 37.0 & 1080 & $320 \times 320$ \\
\hline
\end{tabular}

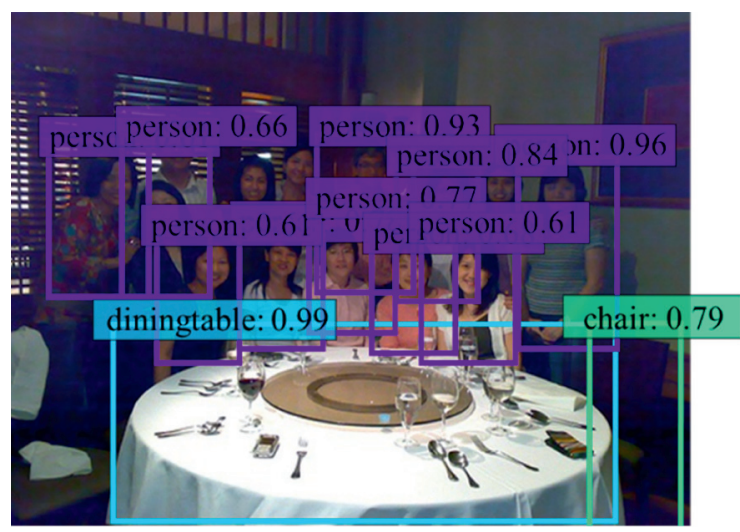

(a)

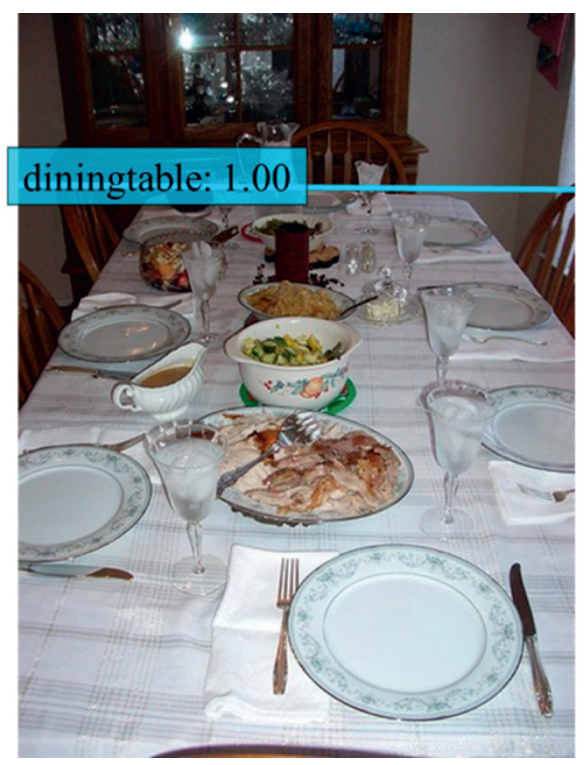

(c)

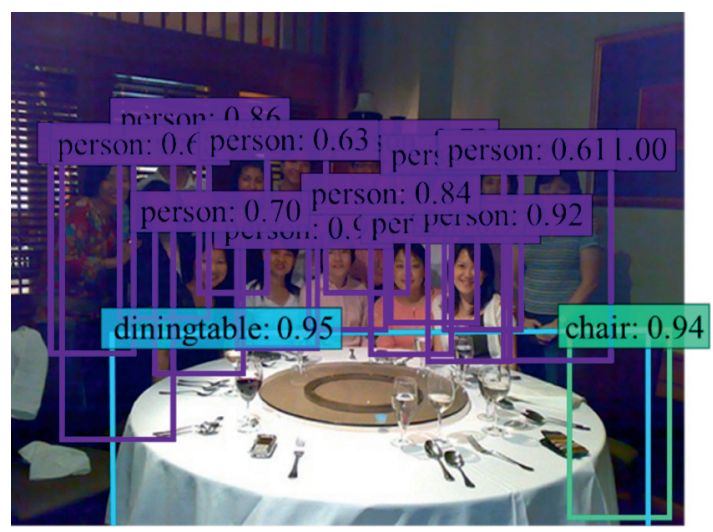

(b)

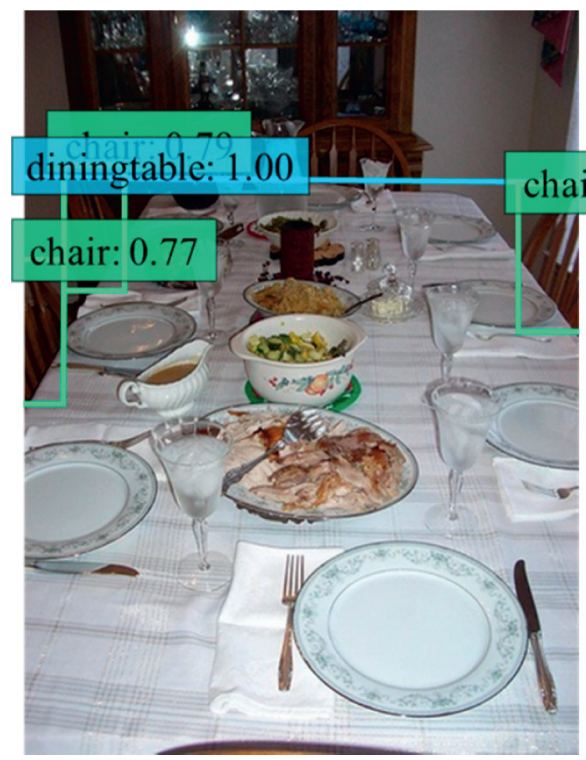

(d)

FIGURE 15: Continued. 


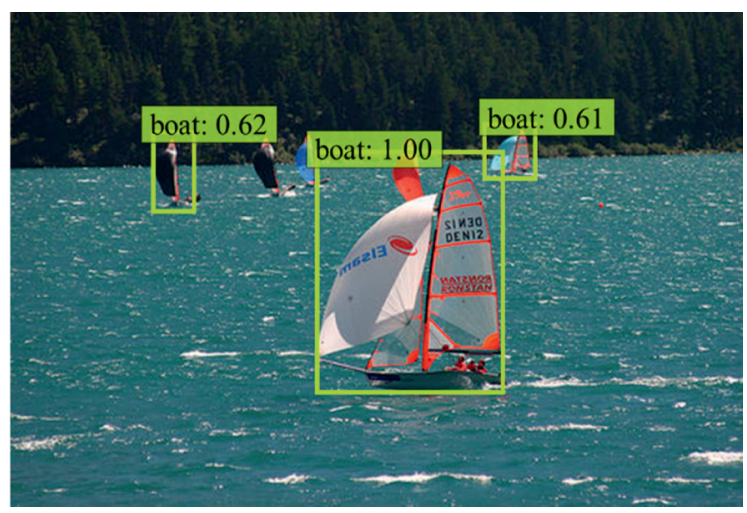

(e)

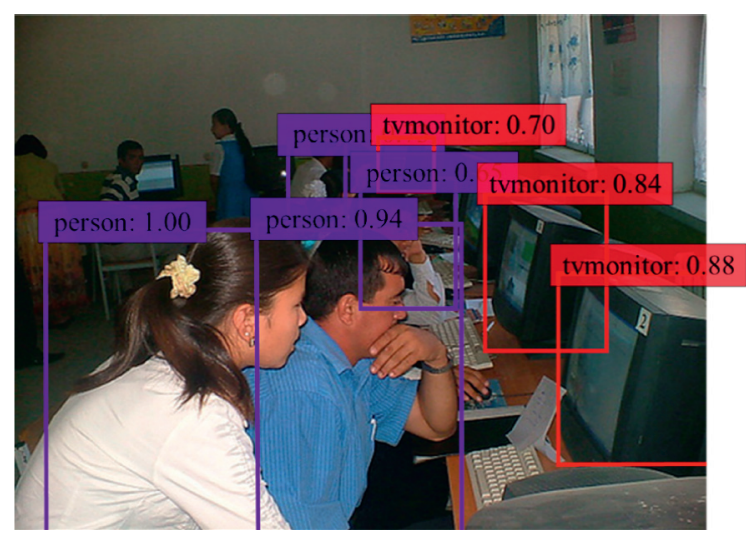

(g)

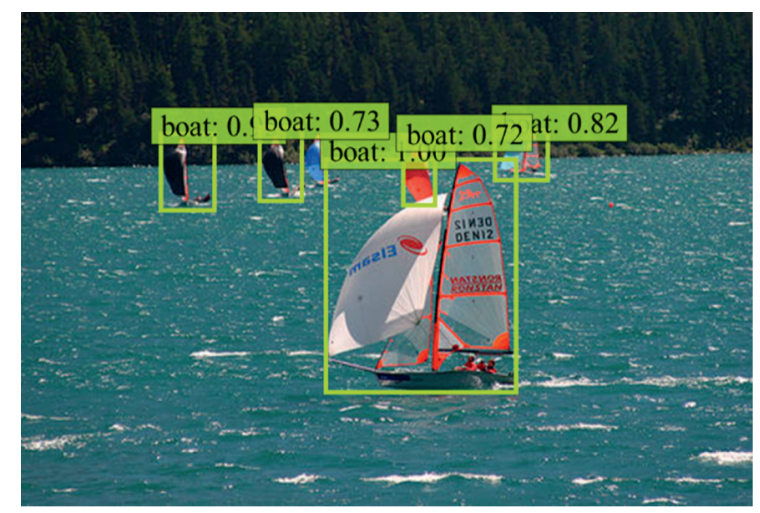

(f)

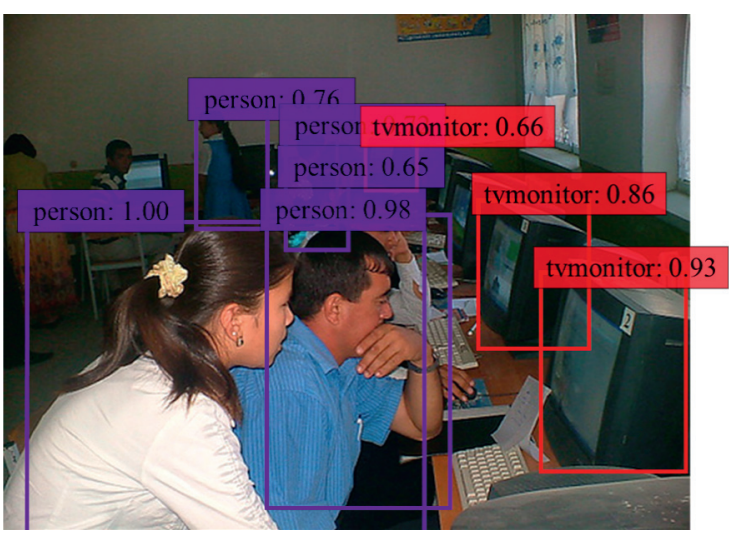

(h)

FIGURE 15: Comparison of detection performances of SSD and SKIPSSD models on some test samples. The first column shows the results of SSD, and the second column is the results of SKIPSSD.

From the data of the first and third rows in Table 4, it could be concluded that in the same network structure, the eltsum fusion method provides better accuracy than concat does. Comparing the second, third, and fourth rows in Table 4, adding BN layers can improve the accuracy of SKIPSSD. When only using BN layers after the eltsum function, SKIPSSD achieves $78.4 \% \mathrm{mAP}, 0.1 \%$ higher than SKIPSSD using BN layers after all convolution layers of the fusion modules. After comprehensive analysis, the SKIPSSD in this paper adopts the eltsum fusion method, and only uses $\mathrm{BN}$ layers in the convolution layer behind eltsum function.

3.3. The Effect of the Upsampling Method on Model Performance. In order to study the effect of upsampling methods on the performance of SKIPSSD, we adopt two kinds of upsampling methods to feature fusion module of SKIPSSD. The first method is deconvolution and dilated convolution, and the specific network structure parameters are shown in Figure 12. The second method is bilinear interpolation, and the specific network structure parameters are shown in Figure 13.

Table 5 shows that SKIPSSD with the bilinear interpolation upsampling method achieves $79.0 \% \mathrm{mAP}$ at $38.7 \mathrm{FPS}$ on the PASCAL VOC2007 test set, outperforming the deconvolution and dilated convolution upsampling method both on speed and accuracy. Therefore, bilinear interpolation is selected as the upsampling method in this paper.

3.4. Effect of the Feature Fusion Structure on Model Performance. This experiment compares the performance on the PASCAL VOC 2007 test set of six different feature fusion structures: SKIPSSD, Part-SKIPSSD, Bi-SKIPSSD, Base-SKIPSSD, AdjacentSSD, and FPNSSD. In this experiment, the upsampling method is bilinear interpolation, the fusion module is $b$, the fusion method is eltsum, and $\mathrm{BN}$ layers are only used in the convolutional layer after eltsum function.

As shown in Table 6, Base-SKIPSSD achieves 78.6\% mAP, $1.4 \%$ higher than SSD by conducting skip connection between layers of VGG base network and prediction layers. However, the lower prediction layers such as Conv4_3_ff still lack enough semantic information for small object detection. FPNSSD adopts a top-down architecture with lateral connections to build high-level semantic feature maps at all scales which is good for multiscale object detection, but fusing features layer by layer is not efficient enough while there are many layers to be combined together. And AdjacentSSD achieves almost the same performance as 
FPNSSD, indicating that there is no need to densely fuse features layer by layer via a top-down architecture. Compared with FPNSSD, by fusing low-level and high-level feature maps skippingly, SKIPSSD is more lightweight and efficient. And the performance of Part-SKIPSSD and BiSKIPSSD demonstrates that less skip connection do not bring obvious advantage of speed, and more skip connections would cause redundancy and do not bring significant accuracy improvement. Thus, in the end, SKIPSSD network is selected as the optimal feature fusion structure.

3.5. Experiments on PASCAL VOC 2007. The loss curve of SKIPSSD is shown in Figure 14(a). Loss keeps decreasing during the training process. In the first 50,000 steps, the loss decreases sharply. After 16,000 steps, the decline speed slows down further, and the loss curve tends to remain unchanged after 200,000 steps. Accordingly, as shown in Figure 14(b), in the first 50,000 steps, the accuracy increases sharply and tends to remain unchanged after 2000 steps, reaching $79.0 \%$ at 22,500 steps.

Table 7 shows the object detection results on the PASCAL VOC 2007 test set. Compared with SSD, SKIPSSD shows a large improvement for 18 classes, including small objects like bottle, boat, bird, plant, and so on, demonstrating that the weakness of small object detection in SSD is improved. With low dimension input $300 \times 300$, SKIPSSD achieves $79.0 \% \mathrm{mAP}$ without bells and whistles, outperforming a lot of state-of-the-art object detection algorithms like Faster R-CNN [3], YOLOv2 [18], YOLOv3 [19], and DSSD [10]. Although the mAP of SKIPSSD is $1 \%$ lower than RefineDet320 [14], RefineDet_SKIP320 achieves $0.4 \%$ mAP higher than RefineDet 320 by adopting skip connection of multiscale feature maps, demonstrating that the skip connection proposed in this paper is effective and can also be integrated into other object detectors.

3.6. Inference Time. Table 8 shows the comparison of speed and accuracy of SKIPSSD and the state-of-the-art object detectors on the PASCAL VOC 2007 test set. For fair comparison, we also test SSD300 [6], RSSD300 [12], and RefineDet320 [14] on the GeForce GTX 1080.

On a single 1080 GPU, SKIPSSD300 achieves $79.0 \%$ $\mathrm{mAP}$ at $38.7 \mathrm{FPS}, 1.8 \% \mathrm{mAP}$ higher than the original SSD and surpassing most of the other state-of-the-art object detection models including two-stage and one-stage methods and other improved SSD models. Although the detection speed of SKIPSSD is a bit slower than SSD due to the extra feature fusion between high-level and low-level features, it is still faster than RSSD [12] and RefineDet [14] and is able to realize real-time detection. And RefineDet_SKIP outperforms RefineDet [14] on both accuracy and speed, demonstrating that skip connection proposed in the paper works better than FPN in the object detection task.

3.7. Visualization. As shown in Figure 15, compared with Figures 15(a) and 15(b), SKIPSSD detects more targets of the same class than SSD when the targets are dense. Compared with Figures 15(c)-15(h), SKIPSSD can detect small objects better than original SSD, and can also "capture" distant objects, which proves that the proposed SKIPSSD based on skip connection of multiscale feature maps can improve the performance of the whole model and the detection performance of small objects.

\section{Conclusions}

In this paper, an improved SSD algorithm SKIPSSD based on skip connection of multiscale feature maps is proposed. In order to fuse high-level and low-level features effectively, a variety of feature fusion modules and fusion connection modules are designed and compared. Experimental results show that with an input size $300 \times 300$ on 1080 GPU, SKIPSSD achieves $79.0 \% \mathrm{mAP}$ at $38.7 \mathrm{FPS}, 1.8 \%$ higher than SSD and can still keep real-time detection speed. In addition, although the skip connection is only adopted to SSD and RefineDet in this paper, it can also be integrated into other object detectors.

In the future work, channel attention mechanism will be adopted to filter out the unimportant channels and improve the saliency of features by learning the importance of each channel.

\section{Data Availability}

The data used to support the findings of this study are available from the corresponding author upon request.

\section{Conflicts of Interest}

The authors declare that there are no conflicts of interest regarding the publication of this paper.

\section{Acknowledgments}

The authors would like to thank the Jiangsu Overseas Visiting Scholar Program for University Prominent Young \& Middle-aged Teachers and Presidents for the support. This work has been supported by the Projects of the National Key Research and Development Plan of China (Grant number: 2016YFB0502103) and the Natural Science Foundation of Jiangsu Province of China (Grant number: BK20160696).

\section{References}

[1] R. Girshick, J. Donahue, T. Darrell, and J. Malik, "Rich feature hierarchies for accurate object detection and semantic segmentation," in Proceedings of the 2014 IEEE Conference on Computer Vision and Pattern Recognition, pp. 580-587, CVPR, Washington, DC, USA, June 2014.

[2] R. Girshick, "Fast R-CNN," in Proceedings of the IEEE International Conference on Computer Vision, pp. 1440-1448, Santiago, Chile, December 2015.

[3] S. Ren, K. He, R. Girshick, and J. Sun, "Faster R-CNN: towards real-time object detection with region proposal networks," in Proceedings of the Advances in Neural Information Processing Systems, pp. 91-99, MIT Press, Montreal, Canada, December 2015. 
[4] J. Dai, L. Yi, K. He et al., "R-fcn: object detection via regionbased fully convolutional networks," in Proceedings of the Advances in Neural Information Processing Systems, pp. 379-387, Barcelona, Spain, December 2016.

[5] J. Redmon, S. Divvala, R. Girshick, and A. Farhadi, "You only look once: unified, real-time object detection," in Proceedings of the IEEE Conference on Computer Vision and Pattern Recognition, pp. 779-788, IEEE Computer Society, Las Vegas, NV, USA, June 2016.

[6] W. Liu, D. Anguelov, D. Erhan et al., "SSD: single shot multibox detector," in Proceedings of European Conference on Computer Vision, pp. 21-37, Springer, Cham, Switzerland, October 2016.

[7] K. He, X. Zhang, S. Ren, and J. Sun, "Spatial pyramid pooling in deep convolutional networks for visual recognition," IEEE Transactions on Pattern Analysis and Machine Intelligence, vol. 37, no. 9, pp. 1904-1916, 2015.

[8] M. Everingham, L. Van Gool, C. K. I. Williams, J. Winn, and A. Zisserman, "The pascal visual object classes (VOC) challenge," International Journal of Computer Vision, vol. 88, no. 2, pp. 303-338, 2010.

[9] Z. Li and F. Zhou, "FSSD: feature fusion single shot multibox detector,” 2017, https://arxiv.org/abs/1712.00960.

[10] C. Y. Fu, W. Liu, A. Ranga et al., "DSSD: deconvolutional single shot detector," 2017, https://arxiv.org/abs/1701.06659.

[11] K. He, X. Zhang, S. Ren et al., "Deep residual learning for image recognition," in Proceedings of IEEE Conference on Computer Vision and Pattern Recognition, pp. 770-778, Seattle, WA, USA, June 2016.

[12] J. Jeong, H. Park, and N. Kwak, "Enhancement of SSD by concatenating feature maps for object detection," 2017, https://arxiv.org/abs/1705.09587.

[13] G. Cao, X. Xie, W. Yang et al., "Feature-fused SSD: fast detection for small objects," in Proceedings of Ninth International Conference on Graphic and Image Processing, pp. 14-16, Qingdao, China, October 2018.

[14] S. Zhang, L. Wen, B. Xiao et al., "Single-shot refinement neural network for object detection," in Proceedings of the IEEE Conference on Computer Vision and Pattern Recognition, pp. 4203-4212, Salt Lake City, UT, USA, June 2018.

[15] Z. Zhang, S. Qiao, C. Xie, W. Shen, B. Wang, and A. L. Yuille, "Single-shot object detection with enriched semantics," in Proceedings of the IEEE Conference on Computer Vision and Pattern Recognition, pp. 5813-5821, Salt Lake City, UT, USA, June 2018.

[16] T. Lin, P. Dollár, R. Girshick et al., "Feature pyramid networks for object detection," 2016, https://arxiv.org/abs/1612.03144.

[17] O. Ronneberger, P. Fischer, and T. Brox, "U-net: convolutional networks for biomedical image segmentation," in Proceedings of the International Conference on Medical Image Computing and Computer-Assisted Intervention, pp. 234-241, Munich, Germany, October 2015.

[18] J. Redmon and A. Farhadi, "YOLO9000: better, faster, stronger," in Proceedings of the IEEE Conference on Computer Vision and Pattern Recognition, pp. 7263-7271, Honolulu, HI, USA, July 2017.

[19] J. Redmon and A. Farhadi, "YOLOv3: an incremental improvement," 2018, https://arxiv.org/abs/1804.02767.

[20] Z. Shen, Z. Liu, J. Li, Y.-G. Jiang, Y. Chen, and X. Xue, "Dsod: learning deeply supervised object detectors from scratch," in Proceedings of the IEEE International Conference on Computer Vision, pp. 1919-1927, Venice, Italy, October 2017. 